\title{
The compressive behaviour and constitutive equation of polyimide foam in wide strain rate and temperature
}

\author{
Akifumi Yoshimoto $^{\text {a }}$, Hidetoshi Kobayashi, Keitaro Horikawa, and Kenichi Tanigaki \\ Grad. Sch. Of Eng. Sci., Osaka University, Toyonaka, Osaka 560-8531, Japan
}

\begin{abstract}
These days, polymer foams, such as polyurethane foam and polystyrene foam, are used in various situations as a thermal insulator or shock absorber. In general, however, their strength is insufficient in high temperature environments because of their low glass transition temperature. Polyimide is a polymer which has a higher glass transition temperature and high strength. Its mechanical properties do not vary greatly, even in low temperature environments. Therefore, polyimide foam is expected to be used in the aerospace industry. Thus, the constitutive equation of polyimide foam that can be applied across a wide range of strain rates and ambient temperature is very useful. In this study, a series of compression tests at various strain rates, from $10^{-3}$ to $10^{3} \mathrm{~s}^{-1}$ were carried out in order to examine the effect of strain rate on the compressive properties of polyimide foam. The flow stress of polyimide foam increased rapidly at dynamic strain rates. The effect of ambient temperature on the properties of polyimide foam was also investigated at temperature from $-190^{\circ} \mathrm{C}$ to $270^{\circ} \mathrm{C}$. The flow stress decreased with increasing temperature.
\end{abstract}

\section{Introduction}

Polymer foams, such as polyethylene foam and polystyrene foam, have excellent shock absorption and thermal insulation performance. Because of their properties they have been used as a shock absorber or thermal insulator in various daily situations $[1,2]$. Therefore, there are many studies focused on constitutive relationships of polymer foams [3-5]. Compressive properties at dynamic strain rates are very important for shock absorbers; therefore, many researchers have studied energy absorption performance of polymer foams at dynamic strain rates by using Split Hopkinson Pressure Bar (SHPB) equipment [6,7]. In general, these foams are suitable enough to absorb impact energy at room temperature. Over $100^{\circ} \mathrm{C}$, however, most polymer foams have insufficient strength as a shock absorber because their glass transition temperature $\left(T_{g}\right)$ is lower than $100^{\circ} \mathrm{C}$.

Polymer foam made of polymer that has a higher $T_{g}$, such as polyimide, is suitable for use at high temperatures. Polyimide has a higher $T_{g}\left(>300^{\circ} \mathrm{C}\right)$ and its mechanical properties do not vary greatly, even in low temperature environments. Therefore, polyimide foam is likely to be used in the aerospace industry and other fields [8]. The mechanical properties of polyimide foam filled honeycombs and properties of standalone polyimide foam at elevated temperatures were also examined $[9,10]$. Thus, determining the constitutive relationship of polyimide foam that can be applied across a wide range of strain rates and temperatures is very useful.

In this study, the effects of strain rate and temperature on the constitutive relationship of polyimide foam were experimentally examined by carrying out a series of

\footnotetext{
${ }^{\text {a }}$ Corresponding author: akifumi . yoshimoto@impact .me.es . osaka-u.ac.jp
}

compression tests. We performed compression tests at various strain rates $\left(10^{-3} \sim 10^{3} \mathrm{~s}^{-1}\right)$ using three testing devices. The effect of temperature was investigated via high or low temperature tests using an electric furnace and liquid nitrogen.

\section{Experiment}

\subsection{Materials and specimen}

Polyimide foam specimen is made by being expanded a volume of polyimide to about hundred times firstly. Density of this foam is approximately $12 \mathrm{~kg} / \mathrm{m}^{3}$. By compressing this foam in one direction while applying heat, two types of polyimide foam specimens were made. In this molding process, open-cell structure with small pieces of thin sheets and short fibers, shown in Fig. 1, piled up in the compressive direction. Because of this structure, polyimide foam specimens in this study show pronounced anisotropy on the mechanical properties. Here, the direction in which small pieces (sheets and fibers) piled up was defined as the $\mathrm{z}$-direction. The normal directions to the $\mathrm{z}$-direction were defined as the $\mathrm{x}$ - and $\mathrm{y}$-directions. Two kinds of polyimide foam specimen with different densities of 120 and $240 \mathrm{~kg} / \mathrm{m} 3$ were provided by Industrial Summit Technology Co., Ltd. (I.S.T.). They have two different foaming magnifications of 10 and 5, denoted by Foam-A and Foam-B, respectively. We prepared cubic specimens with $20 \mathrm{~mm}$ sides. These specimens geometries are shown in Fig. 2(a).

To investigate the strain rate and temperature dependence of polyimide itself, the same base material used in the foam specimens, we prepared additional, bulk polyimide specimens. That specimen geometry is shown in Fig. 2(b). This second specimen was a cylindrical

This is an Open Access article distributed under the terms of the Creative Commons Attribution License 4.0, which permits unrestricted use, distribution, and reproduction in any medium, provided the original work is properly cited. 


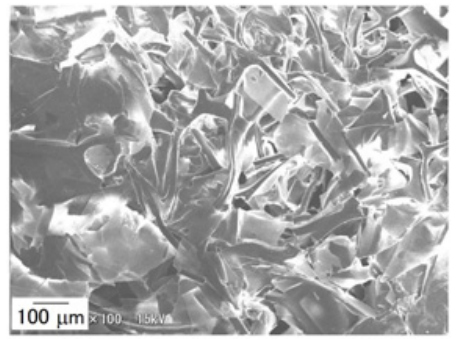

Figure 1. Microstructure of polyimide foam.

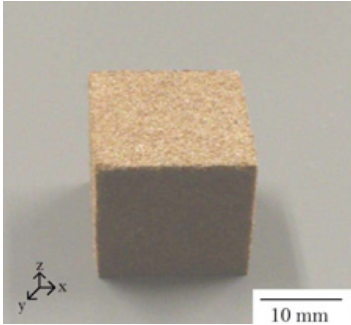

(a)

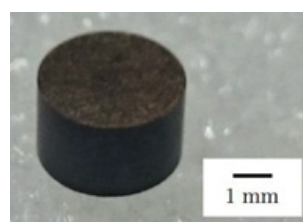

(b)
Figure 2. Specimen for compression tests. (a): Polyimide foam (b): Bulk-specimen.

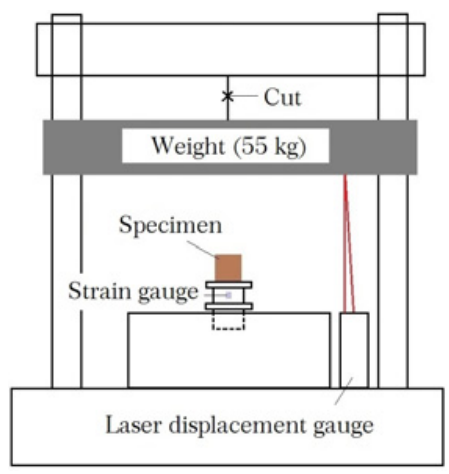

Figure 3. Dropping weight testing machine.

with a diameter of $5 \mathrm{~mm}$ and a height of $3 \mathrm{~mm}$. This specimen is molded in the same way as the foam specimen. Compressive deformation is added until its density reached the same as polyimide.

\subsection{Compression tests}

By using a universal testing machine (Shimazu autograph), quasi-static compression tests were carried out. The strain rates evaluated were $1.7 \times 10^{-3}$ and $1.7 \times 10^{-1} \mathrm{~s}^{-1}$. In high temperature quasi-static tests at $200^{\circ} \mathrm{C}$ and $270^{\circ} \mathrm{C}$, specimens was heated to a desired temperature by an electric furnace. These temperature were determined within a range that does not exceed the glass-transition temperature $T_{g}\left(=310^{\circ} \mathrm{C}\right)$ of polyimide foam. The heated specimen was compressed after being kept in the furnace at the desired temperature for fifteen minutes. In low temperature quasi-static tests, specimens were cooled to $-190^{\circ} \mathrm{C}$ by liquid nitrogen in a polystyrene foam box attached to the apparatus. Similarly, after maintaining the target temperature for fifteen minutes, the specimen was compressed. The same compression tests were also carried out on the Bulk-specimen.

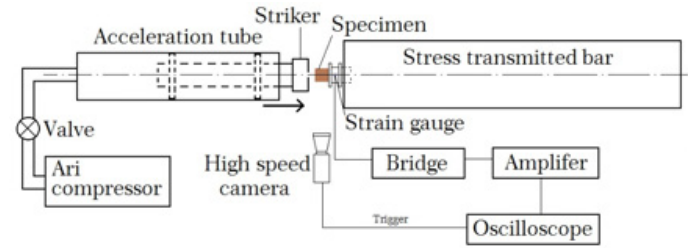

(a)

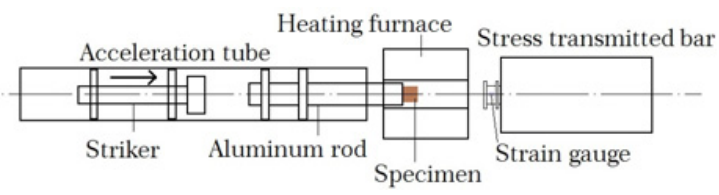

(b)

Figure 4. Dynamic test apparatus. (a): room temperature test (b): high temperature test.

A dropping weight testing machine, shown in Fig. 3, was used in intermediate speed tests. A dropping weight of $55 \mathrm{~kg}$ falls along a rigid frame and compresses the specimen. Its strain rate is approximately $50 \mathrm{~s}^{-1}$. The deformation of the specimen was measured by laser displacement gauge. Load was measured by a special load cell, that contains a small detective section and stress transmitted block. This method when used in dynamic tests takes a relatively long time to apply the load to the specimen [11].

The dynamic compression test apparatus is shown in Fig. 4(a). A striker in an acceleration tube is fired by compressed air and impacts on the specimen which is attached to the special load cell. Its strain rate is about $1.0 \times 10^{3} \mathrm{~s}^{-1}$. The load was measured by the same method as that used in the dropping weight tests. The deformation of the specimen was measured by using a high-speed video camera (Phantom V4.2) with 25,000 frames per second.

Dynamic tests were carried out at high and low temperatures as well as at room temperature. Test temperatures were room temperature, $200^{\circ} \mathrm{C}, 270^{\circ} \mathrm{C}$ and $-190^{\circ} \mathrm{C}$. The setup for dynamic tests at high temperature is shown in Fig. 4(b). Specimens were attached to the aluminum rod and heated to the desired temperature by an electric furnace and fired by impacting with a striker.

Its strain rate is about $250 \mathrm{~s}^{-1}$. This method was adopted to avoid the elevated temperatures contacting the semiconductor strain gauge on the load cell. Low temperature dynamic tests were carried out by using the same apparatus. Before the test, specimens were cooled in a refrigerant box next to the testing machine. After a specimen was removed from the refrigerant box, it was attached to the aluminum rod immediately and compressed by striking the load cell.

This setup is used for tests that required a relatively long time to deformation and it is not suitable for small specimen like the Bulk-specimen because of the limited resolution of the high-speed video camera. Therefore, a Split Hopkinson Pressure Bar (SHPB) apparatus, as shown in Fig. 5, was used in dynamic tests for Bulk-specimens. The striker, input bar and output bar are made of SUS304 with a diameter of $30 \mathrm{~mm}$. The nominal stress $\sigma_{n}$, nominal strain $\varepsilon_{n}$ and strain rate $\dot{\varepsilon}$ relationship of the specimen in 


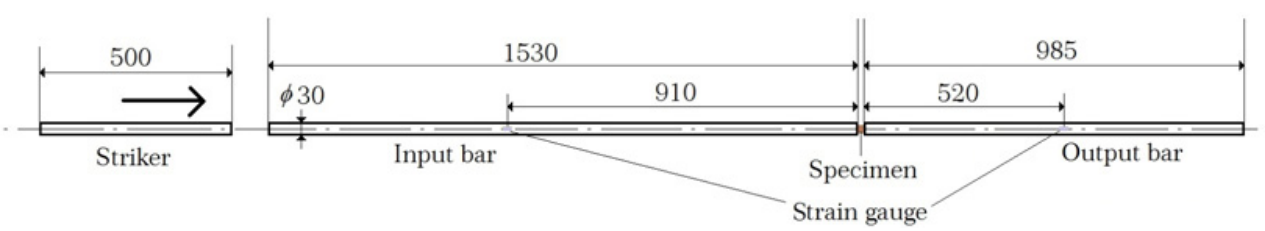

Figure 5. Scematic of SHPB apparatus.

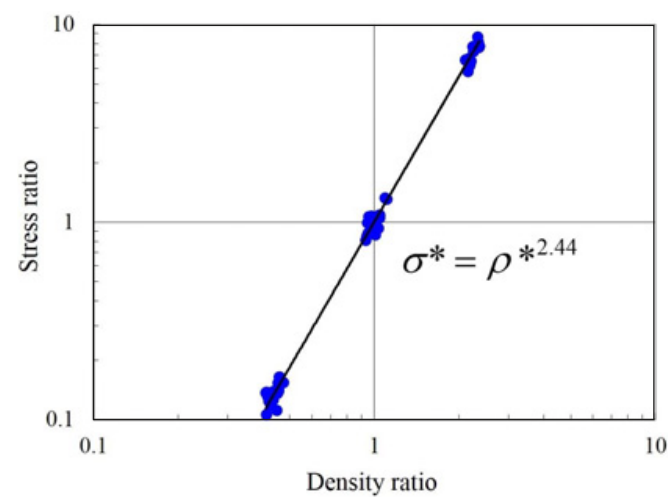

Figure 6. Relationship between strass ratio and density ratio.

SHPB is as follows

$$
\begin{gathered}
\sigma_{m}=\frac{A \sigma_{t}}{A_{s}} \\
\dot{\varepsilon}=\frac{2}{\rho C L_{s}}\left(\sigma_{i}-\sigma_{t}\right) \\
\varepsilon_{n}=\frac{2}{\rho C L_{s}} \int\left(\sigma_{i}-\sigma_{t}\right) d t .
\end{gathered}
$$

Where, $\sigma_{i}, \sigma_{t}$ and $\sigma_{r}$ are the incident pulse, transmitted pulse and reflected pulse respectively. $A, C$ and $\rho$ are the cross-sectional area, the velocity of elastic wave and density of the input and output bar respectively. $A_{s}$ and $L_{s}$ are the cross-sectional area and height of specimen respectively.

\section{Experimental results and discussions}

\subsection{Density normalization of specimen}

Generally, compressive strength of foam material is sensitive to its relative density [12]. The polyimide foam specimens in this study have individual differences in the density. Therefore, there are differences in the constitutive relationship for the same specimen type. To evaluate the effects of strain rate and temperature it is necessary to control for the effect of density. We defined two ratios: one is the ratio of specimen density, $\rho^{*}\left(=\rho_{1} / \rho_{2}\right)$, and the other is the ratio of average stress, $\sigma^{*}\left(=\sigma_{1} / \sigma_{2}\right)$, where the subscripts of 1 and 2 indicate two arbitrary specimens compressed under the same conditions. Here, the average stress was defined as the mean stress in the range of 0.2 to 0.4 strain. Data plots of $\rho^{*}$ and $\sigma^{*}$ is shown in Fig. 6 . From this graph we determined the equation expressing the relationship between $\rho^{*}$ and $\sigma^{*}$ as follows

$$
\sigma *=\rho *^{2.44} \text {. }
$$

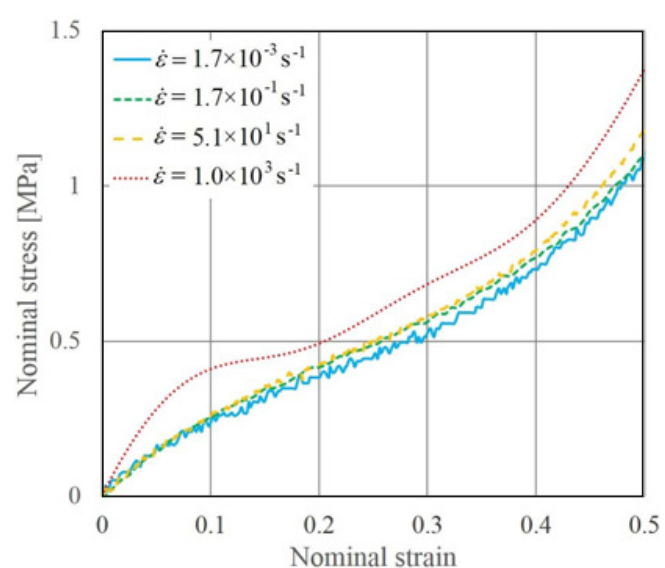

(a)

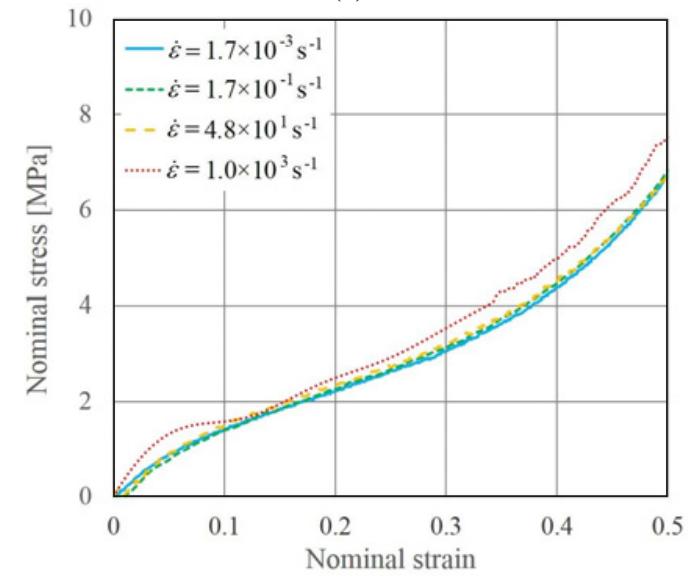

(b)

Figure 7. Stress-strain curves at various strain rates at room temperature. (a): Foam-A (b): Foam-B.

Experimental data of specimens that include the difference in density are normalized with the nominal density of Foam-A $\left(120 \mathrm{~kg} / \mathrm{m}^{3}\right)$ and Foam-B $\left(240 \mathrm{~kg} / \mathrm{m}^{3}\right)$. By doing so, it is possible to cancel the effect of density. In the following, we discuss based on the normalized data.

\subsection{Strain rate dependence}

Because of specimen anisotropy, its mechanical response indicates significant differences depending on the compression direction. If a specimen is compressed in the $\mathrm{X}$ - or y-directions, it cracks at about strain $\varepsilon=0.3$ (Foam-A) or 0.1 (Foam-B). Specimens do not crack when compressed in the $\mathrm{z}$ - direction, and it is possible to be compressed into the densification region. Therefore, we focused on compressive strength in $\mathrm{z}$-direction in this study.

The stress-strain curves of Foam-A and -B obtained from compression tests at various strain rates at room temperature are shown in Fig. 7. 


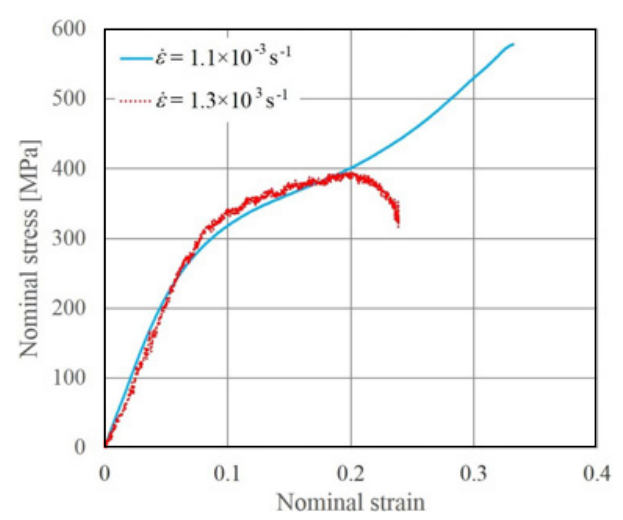

Figure 8. Stress-strain curves of Bulk-specimens at quasi-static and dynamic strain ratess at room temperature.

Generally, compressive behaviour of polymer foam consists of three stages: liner elastic, plateau and densification regions. Polyimide foam in this study also indicates roughly the same behaviour, but its liner elastic and plateau regions are unclear. In both Foam-A and -B, in the range of strain rate from quasi-static to intermediate, compressive flow stress has little difference. However compressive flow stress at dynamic strain rates is greater than others. This stress increment is sufficiently larger than the variation due to individual difference of specimen. Therefore, it can be said that the strain rate dependence of polyimide foam does not affect the compressive property up to the intermediate strain rate $\left(\dot{\varepsilon} \approx 50 \mathrm{~s}^{-1}\right)$. And above intermediate strain rates the compressive strength of polyimide foam indicates salient strain rate dependence. It is considered that this dependence is related to the polyimide itself and the foam structure. Stress-strain curves of Bulk-specimens at quasi-static and dynamic strain rates are shown in Fig. 8. The differences due to strain rate are not observed in these graphs. This result indicates polyimide itself does not have strain rates dependence at strain rates from $10^{-3}$ to $10^{3} \mathrm{~s}^{-1}$. Therefore, we can say that the effect of strain rate of compressive property of polyimide foam is related to the foam structure.

\subsection{Temperature dependence}

The stress-strain curves of Foam-A and -B obtained from compression tests at various temperatures at quasistatic strain rates are shown in Fig. 9. It is found that the compressive flow stress decreases with increasing temperature. These graphs indicate that compressive strength of polyimide foam has salient temperature dependence. Especially at $270^{\circ} \mathrm{C}$, the effect of temperature is appeared to be strong. The graph does not have an upward convex shape in the early region unlike others. At $-190^{\circ} \mathrm{C}$, flow stress increases significantly and some Foam-A specimens cracked in the compression process. It is found that polyimide foam becomes brittle near the temperature of liquid nitrogen at quasi-static strain rates.

The stress-strain curves of Foam-A and -B obtained from compression tests at various temperatures at dynamic strain rates $\left(\dot{\varepsilon} \approx 250 \mathrm{~s}^{-1}\right)$ are shown in Fig. 10.

At $-190^{\circ} \mathrm{C}$, Foam-A specimen fractured, similar to quasi-static tests. Figure 11 shows the specimen after

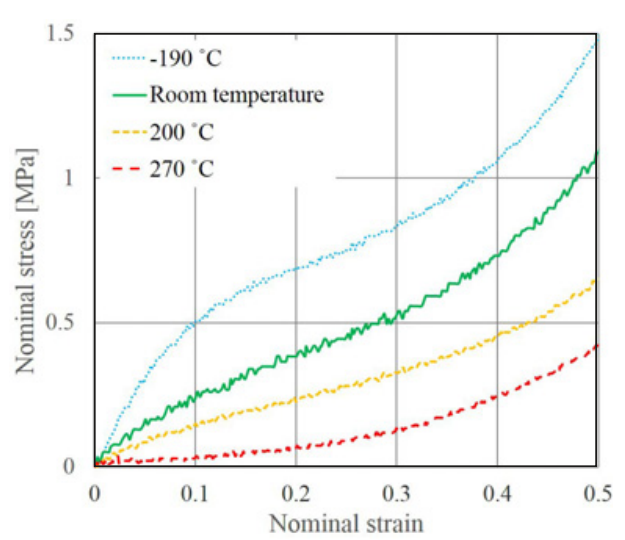

(a)

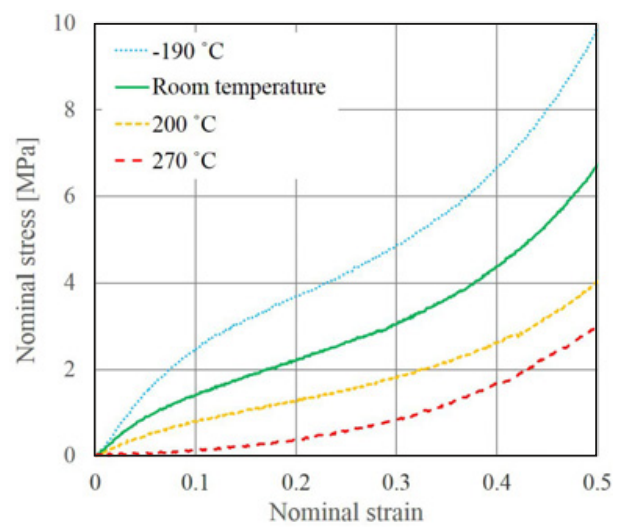

(b)

Figure 9. Stress-strain curves at various temperatures at quasistatic strain rates. (a): Foam-A (b): Foam-B.

compression tests. These results indicate that polyimide foam becomes brittle near the liquid nitrogen temperature at any strain rate. In dynamic tests, the effect of low temperature is less than that observed during quasi-static tests. The cause of this behaviour is that flow stress decreased due to temperature rise that accompanied strain rate. Flow stress of Foam-A at $-190{ }^{\circ} \mathrm{C}$ became lower than the result at room temperature over strain 0.3 . It is considered that this is caused by fracture and crushing during compression tests of Foam-A.

The stress-strain curves of Bulk-specimen at various temperatures are shown in Fig. 12. These graphs indicate that polyimide itself has significant temperature dependence. Therefore, it is considered that temperature dependence of polyimide foam is attributed to the polyimide material.

\subsection{Constitutive equation}

Based on these results, we devised constitutive equations of polyimide foam. However, the applicable range was limited to above room temperature. Results of low temperature tests may not be the correct since specimen cracked during compression tests at $-190^{\circ} \mathrm{C}$. From the obtained stress-strain curves, the initial region of compressive deformation is expressed by the following equation.

$$
\sigma=a(1-\exp (-c \varepsilon))
$$




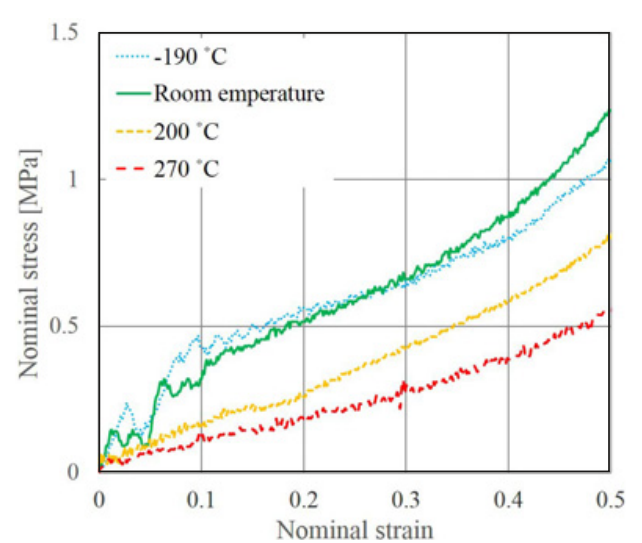

(a)

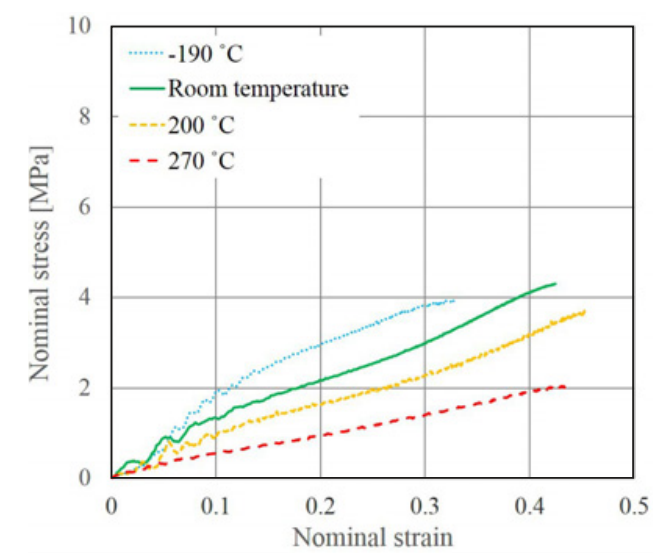

(b)

Figure 10. Stress-strain curves at various temperatures at dynamic strain rates. (a): Foam-A (b): Foam-B.

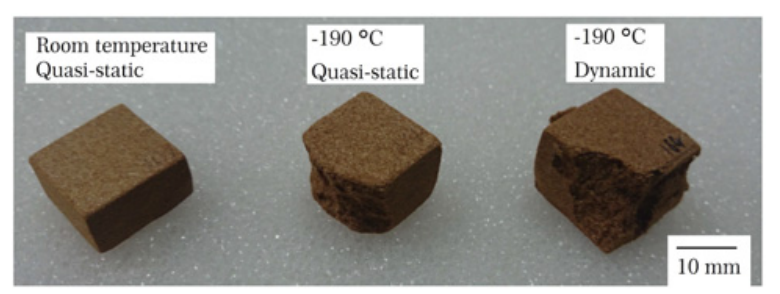

Figure 11. Specimens after compression tests.

In the study focused on the constitutive equation in initial region of PMMA foam, similar expression is used [13]. Where, $a$ is constant. From the results of compression tests of polyimide foam, it is found that strain rate and temperature affect the initial gradient of stress-strain curves strongly. Therefore, in Eq. (5), we defined $c$ as a variable of strain rate and temperature. Considering the test results, we expressed $c$ in the variable which varies greatly in dynamic strain rate. The effect of temperature could be well expressed in the linear relationship. However, the effect of temperature on $c$ varies by strain rate. Therefore, the function of strain rate described above is incorporated. To summarize these, variable $c$ is expressed by the following equation.

$$
c(\dot{\varepsilon}, T)=c_{0}\left(1+m \frac{\dot{\varepsilon}}{\dot{\varepsilon}_{0}}\right)\left(1-p \frac{T}{1+q\left(\dot{\varepsilon} / \dot{\varepsilon}_{0}\right)}\right) .
$$

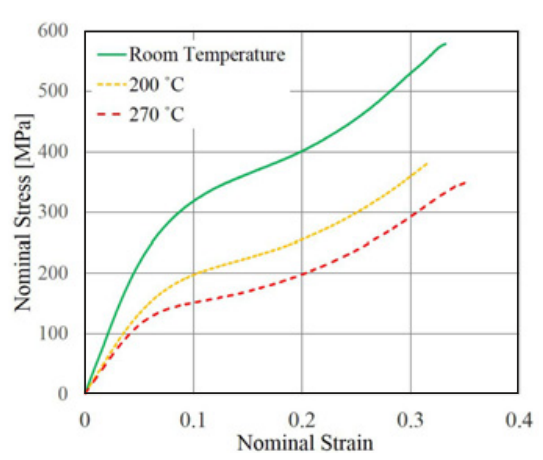

Figure 12. Stress-strain curves of Bulk-specimens at various temperatures at quasi-static strain rates.

Where, $c_{0} . m, p$ and $q$ are constant. $\dot{\varepsilon}_{0}$ is optional reference strain rate. In this case $\dot{\varepsilon}_{0}=0.001 . \dot{\varepsilon}$ and $T$ are strain rate and absolute temperature respectively.

Late region of compression is suitably expressed by an exponential function because the flow stress increases rapidly after densification. Therefore, the late region is expressed by the following equation.

$$
\sigma=b \exp (d \varepsilon)
$$

Where, $b$ is constant. From the test results, varying the strain rate and temperature affects the starting point of densification. Thus, we defined that $d$ is a variable of strain rate and temperature. Since the effects of strain rate and temperature on late regions of stress-strain curves are almost the same as the effects on the initial gradient, $d$ could be expressed by nearly the same equation as $c$. However, the effect of temperature on $d$ does not vary by strain rate, $d$ is expressed by a more simple equation than $c$. Therefore, variable $d$ is expressed by the following equation.

$$
d(\dot{\varepsilon}, T)=d_{0}\left(1+r \frac{\dot{\varepsilon}}{\dot{\varepsilon}_{0}}\right)(1-s T) .
$$

Where, $d_{0} . r$ and $s$ are constant.

As a result, we expressed the constitutive equation of polyimide foam by the following equation, it is sum of Eqs. (5) and (7).

$$
\sigma=a(1-\exp (-c \varepsilon))+b \exp (d \varepsilon)
$$

We calculated the variation of the flow stress at each strain rate and temperature. Based on those results the constants were determined by trial and error. The determined constants of Foam-A and -B are shown in Table 1. Results of calculating the constitutive relationship from Eq. (9) and experimental results at various strain rates at room temperature of Foam-A are shown in Fig. 13. Error between the experimental results and the calculated constitutive relationship at room temperature of Foam-A is shown in Fig. 14. In the region that indicates low stress, the error rate is larger, however, it is found that these results are consistent.

The same graphs of Foam-A at dynamic strain rates $\left(\dot{\varepsilon} \approx 250 \mathrm{~s}^{-1}\right)$ at various temperatures are shown in Figs. 15 and 16. In these plots, experimental results 
Table 1. Constants of constitutive equations of polyimide FoamA and $-\mathrm{B}$.

\begin{tabular}{|c|c|c|}
\hline & Foam-A & Foam-B \\
\hline$a$ & 0.73 & 2.9 \\
\hline$b$ & $5.8 \times 10^{-3}$ & $8.4 \times 10^{-2}$ \\
\hline$c_{0}$ & 7.7 & 12 \\
\hline$d_{0}$ & 10 & 9.5 \\
\hline$m$ & $7.8 \times 10^{-7}$ & $1.7 \times 10^{-7}$ \\
\hline$p$ & $1.76 \times 10^{-3}$ & $1.81 \times 10^{-3}$ \\
\hline$q$ & $3.0 \times 10^{-7}$ & $2.7 \times 10^{-7}$ \\
\hline$r$ & $7.1 \times 10^{-8}$ & $2.6 \times 10^{-8}$ \\
\hline$s$ & $4.1 \times 10^{-4}$ & $5.6 \times 10^{-4}$ \\
\hline
\end{tabular}

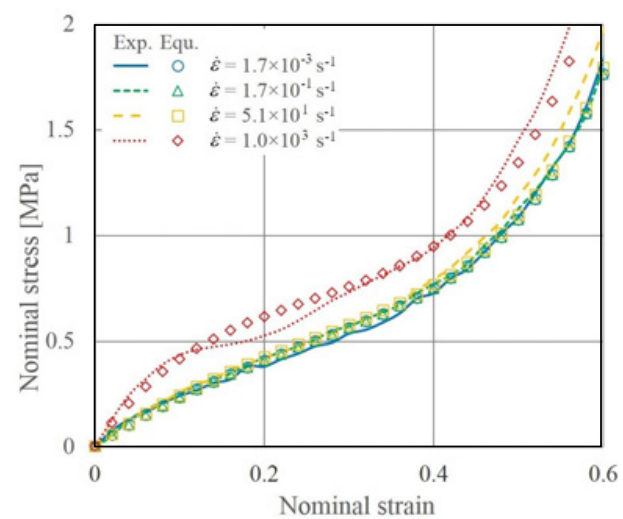

Figure 13. Experimental results and constitutive equations at room temperature of Foam-A.

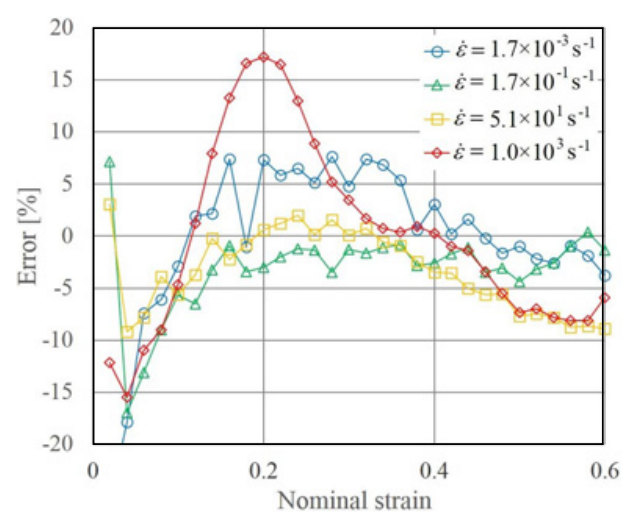

Figure 14. Error between the experimental results and the constitutive equations at room temperature of Foam-A.

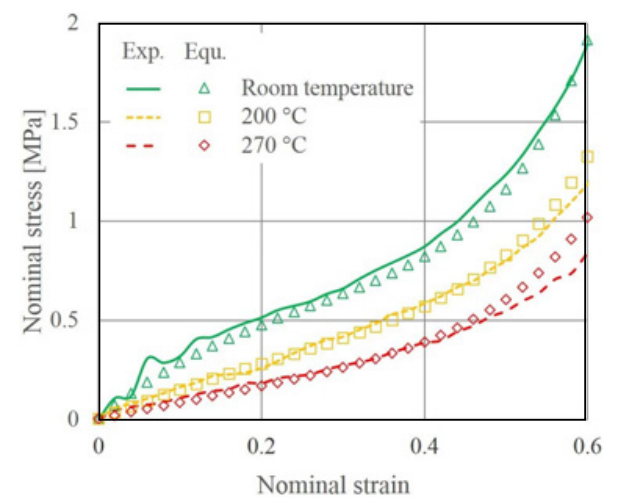

Figure 15. Experimental results and constitutive equations at dynamic strain rates of Foam-A.

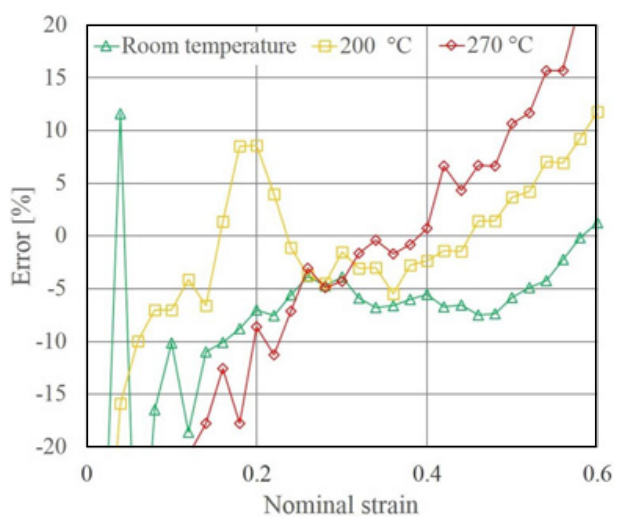

Figure 16. Error between the experimental results and the constitutive equations at dynamic strain rates of Foam-A.

and calculated values are found to be consistent. When evaluating all results, including results of quasi-static strain rates and Foam-B, this constitutive equation can express the constitutive relationship of polyimide foam with an error within $\pm 15 \%$ at strain $\varepsilon<0.6$.

\section{Conclusion}

The effects of strain rate and temperature on the compressive property of polyimide foam were investigated by a series of compression tests. The principal results obtained are as follows.

1) The flow stress during compressive deformation of polyimide foam indicates salient strain rate dependence above intermediate strain rates.

2) The flow stress during compressive deformation of polyimide foam indicates salient temperature dependence.

3) Over room temperature, the constitutive equation using an exponential function can express the constitutive relationship during compressive deformation of polyimide foam with an error of within $\pm 15 \%$ at a strain $\varepsilon<0.6$.

We would like to express our grateful appreciation to Mr. T. Sakata and I.S.T. Co. Ltd. for the provision of the polyimide foams.

\section{References}

[1] L. Sorrentino, M. Aurilia, S. Iannace, Polymer Testing, 26, 878 (2007)

[2] P. Viot, F. Beani, J. Lataillade, J. Mat. Sci., 40, 5829 (2005)

[3] Q. Liu, G. Subhash, Polymer Eng. Sci., 44, 463 (2004)

[4] S. Ouellet, D. Cronin, M. Worswick: Polymer Testing, 25, 731 (2006)

[5] R. Bouix, P. Viot, J.L. Lataillade, Int. J. Impact Eng., 36, 329 (2009)

[6] B. Song, W. Chen, S. Dou, N. Winfree, J. Kang Int. J. Impact Eng., 31, 509 (2005)

[7] J. Yi, M. Boyce, G. Lee, E. Balizer, Polymer, 47, 319 (2006) 
[8] Y. Takeda. T. Hakamada, N. Hama, K. Moriuchi: preprinted Coll. 3D10(JSASS-2009-5137)

[9] C. Resewski, W. Buchgraber, Materialwissen-schaft und Werkstofftechnik, 34, 364 (2003)

[10] A. Kuwabara, M. Ozasa, T. Shimokawa, N. Watanabe and K. Nomoto, Adv. Compo. Mater., 14, 343 (2005)
[11] S. Tanimura: Soc. of Mech. Eng. Coll. of papers A 68, 1767 (2002)

[12] A. Gibson, M. Ashby, CELLULAR SOLIDS: Structure \& Properties (1999)

[13] C. Jo, J. Fu, H. Naguib, Polymer, 46, 11896 (2005) 